\title{
An integrated sustainable manufacturing strategy framework using fuzzy analytic network process
}

\author{
Ocampo, L.A. ${ }^{\mathrm{a},{ }^{*}}$, Clark, E.E. ${ }^{\mathrm{b}}$, Tanudtanud, K.V.G. ${ }^{\mathrm{c}}$, Ocampo, C.O.V. ${ }^{\mathrm{d}}$, Impas Sr., C.G. ${ }^{\mathrm{a}}$, Vergara, V.G. ${ }^{\mathrm{a}}$, \\ Pastoril, J. ${ }^{a}$, Tordillo, J.A.S. ${ }^{a}$ \\ ${ }^{a}$ University of San Carlos, Department of Mechanical Engineering, Cebu City, Cebu, Philippines \\ ${ }^{b}$ De La Salle University, Department of Industrial Engineering, 2401 Taft Avenue, Manila, Philippines \\ 'International Society for Business Innovation \& Technology Management (ISBITM), Radium St., Manila, Philippines \\ dUniversity of San Carlos, Department of Industrial Engineering, Cebu City, Cebu, Philippines
}

\begin{abstract}
A B S T R A C T
This paper adopts a fuzzy analytic network process approach for developing a sustainable manufacturing strategy under the influence of stakeholders' interests. Frameworks developed in literature tend to structure manufacturing strategy in such a way that addresses market needs and expectations. As the move towards sustainability becomes highly pronounced, literature in domain manufacturing is developing approaches and initiatives that explore different facets of sustainability. However as this impetus becomes increasingly famous, manufacturing firms are faced with the challenge of integrating sustainability with the classical function of manufacturing, which is to support firms' competitive advantages. Thus, an inclusive approach would constitute a manufacturing strategy that would support not only sustainability but enhance the competitive strategy of a firm. In order to integrate these two objectives it is necessary to take into consideration the different stakeholders' interests as significant drivers towards sustainability. This work explores the significance of these interests when developing a manufacturing strategy using the proposed approach. In the proposed method, an analytic network process handles the complexity of the decision framework, and judgment elicitation during pairwise comparisons is described using linguistic variables with equivalent triangular fuzzy numbers. The proposed approach is useful when handling complexity and uncertainty especially in group decisionmaking. The content of the sustainable manufacturing strategy using a fuzzy analytic process is presented in this paper.
\end{abstract}

\section{ARTICLE INFO}

Keywords:

Manufacturing strategy

Sustainability

Uncertainty

Analytic network process

Fuzzy set theory

*Corresponding author: don_leafriser@yahoo.com (Ocampo, Lanndon A.)

Article history:

Received 20 May 2015

Revised 12 August 2015

Accepted 19 August 2015 


\title{
Integrirana strategija trajnostne proizvodnje $z$ uporabo analitičnega mrežnega procesa
}

\author{
Ocampo, L.A. ${ }^{\mathrm{a},{ }^{*}}$, Clark, E.E. ${ }^{\mathrm{b}}$, Tanudtanud, K.V.G. ${ }^{c}$, Ocampo, C.O.V. ${ }^{\mathrm{d}}$, Impas Sr., C.G. ${ }^{\mathrm{a}}$, Vergara, V.G. ${ }^{\mathrm{a}}$, \\ Pastoril, J. ${ }^{a}$, Tordillo, J.A.S. ${ }^{a}$ \\ ${ }^{a}$ University of San Carlos, Department of Mechanical Engineering, Cebu City, Cebu, Philippines \\ ${ }^{b}$ De La Salle University, Department of Industrial Engineering, 2401 Taft Avenue, Manila, Philippines \\ 'International Society for Business Innovation \& Technology Management (ISBITM), Radium St., Manila, Philippines \\ dUniversity of San Carlos, Department of Industrial Engineering, Cebu City, Cebu, Philippines
}

\section{POVZETEK}

Raziskava je bila namenjena prilagoditvi mehkega analitičnega mrežnega procesa za razvoj strategij trajnostne proizvodnje pod vplivom interesov vpletenih deležnikov. Iz literature je mogoče razbrati, da se proizvodne strategije poskuša strukturirati tako, da zadostijo pričakovanjem in potrebam trga. Ker je trajnostni razvoj vedno bolj zaželen, je mogoče zaslediti čedalje več razvojnih pristopov in pobud, ki raziskujejo različne vidike sonaravne proizvodnje. Podjetja morajo zato poleg izvajanja utečenega proizvodnega postopka vgraditi v svoj razvoj tudi načela trajnostnega razvoja, kar prispeva k njihovim konkurenčnim prednostim. Takšen celosten pristop torej ne vključuje le trajnostnega razvoja podjetja, ampak izboljšuje njegovo strategijo konkurenčnosti. Da bi spojili ta dva cilja je treba upoštevati interese različnih deležnikov. To delo raziskuje pomembnost teh interesov pri razvoju proizvo$\mathrm{dnih}$ strategij $\mathrm{z}$ uporabo predlaganega pristopa. $\mathrm{V}$ predlaganem pristopu analitični mrežni proces obvladuje kompleksne odvisne povezave odločitvenega problema, medtem ko teorija mehkih množic obravnava nejasnost individualne presoje. Predlagan pristop je koristen, ko je treba obvladovati zapletenost in negotovost, še posebej v skupinskem odločanju.

\section{PODATKI O ČLANKU}

Ključne besede: Proizvodna strategija

Trajnostni razvoj

Negotovost Analitični mrežni proces

Teorija mehkih množic

*Kontaktna oseba: don_leafriser@yahoo.com (Ocampo, Lanndon A.)

Zgodovina članka:

Prejet 20. maja 2015

Popravljen 12. avgusta 2015

Sprejet 19. avgusta 2015 


\section{References}

[1] Skinner, W. (1969). Manufacturing - missing link in corporate strategy, Harvard Business Review, Vol. 47, No. 3, 136-145.

[2] Wheelwright, S.C. (1984). Manufacturing strategy: Defining the missing link, Strategic Management Journal, Vol. 5, No. 1, 77-91, doi: 10.1002/smj.4250050106.

[3] Fine, C.H., Hax, A.C. (1985). Manufacturing strategy: A methodology and an illustration, Interfaces, Vol. 15, No. 6, 28-46, doi: 10.1287/inte.15.6.28.

[4] Hayes, R.H., Pisano, G.P. (1996). Manufacturing strategy: At the intersection of two paradigm shifts, Production and Operations Management, Vol. 5, No. 1, 25-41, doi: 10.1111/j.1937-5956.1996.tb00383.x.

[5] Paloviita, A., Luoma-aho, V. (2010). Recognizing definitive stakeholders in corporate environmental management, Management Research Review, Vol. 33, No. 4, 306-316, doi: 10.1108/01409171011030435.

[6] Kassinis, G., Vafeas, N. (2006). Stakeholder pressures and environmental performance, Academy of Management Journal, Vol. 49, No. 1, 145-159, doi: 10.5465/AMJ.2006.20785799.

[7] Baden, D.A., Harwood, I.A., Woodward, D.G. (2009). The effect of buyer pressure on suppliers in SMEs to demonstrate CSR practices: An added incentive or counter productive?, European Management Journal, Vol. 27, No. 6, 429-441, doi: 10.1016/j.emj.2008.10.004.

[8] Ocampo, L.A., Clark, E.E. (2014). Developing a framework for sustainable manufacturing strategies selection, DLSU Business \& Economics Review, Vol. 23, No. 2, 115-131.

[9] Pham, D.T., Thomas, A.J. (2012). Fit manufacturing: A framework for sustainability, Journal of Manufacturing Technology Management, Vol. 23, No. 1, 103-123, doi: 10.1108/17410381211196311.

[10] Ocampo, L.A., Clark, E.E., Tanudtanud, K.V.G. (2015). Structural decisions of sustainable manufacturing strategy with fuzzy analytic network process (FANP), International Journal of Strategic Decision Sciences, Vol. 6, No. 2, 1227, doi: 10.4018/ijsds.2015040102.

[11] Ocampo, L.A., Clark, E.E., Tanudtanud, K.V.G. (2015). A sustainable manufacturing strategy from different strategic responses under uncertainty, Journal of Industrial Engineering, Vol. 2015, 1-11, doi: 10.1155/2015/210568.

[12] Tseng, M.L., Chiu, A.S.F. (2013). Evaluating firm's green supply chain management in linguistic preference, Journal of Cleaner Production, Vol. 40, 22-31, doi: 10.1016/i.jclepro.2010.08.007.

[13] Saaty, T.L. (2001). Decision making with dependence and feedback: The Analytic Network Process, RWS Publications, Pittsburgh.

[14] Ocampo, L.A., Clark, E.E. (2014). A comprehensive evaluation of sustainable manufacturing programs using Analytic Network Process (ANP), Multiple Criteria Decision Making, Vol. 9, 101-122.

[15] Ocampo, L.A. (2015). A hierarchical framework for index computation in sustainable manufacturing, Advances in Production Engineering \& Management, Vol. 10, No. 1, 40-50, doi: 10.14743/apem2015.1.191.

[16] Pan, R., Zhang, W., Yang, S., Xiao, Y. (2014). A state entropy model integrated with BSC and ANP for supplier evaluation and selection, International Journal of Simulation Modelling, Vol. 13, No. 3, 348-363, doi: 10.2507/ ijsimm13(3)co13.

[17] Hayes, R.H., Wheelwright, S.C. (1984). Restoring our competitive edge: Competing through manufacturing, John Wiley \& Sons, New York.

[18] Marucheck, A., Pannesi, R., Anderson, C. (1990). An exploratory study of the manufacturing strategy process in practice, Journal of Operations Management, Vol. 9, No. 1, 101-123, doi: 10.1016/0272-6963(90)90148-7.

[19] Platts, K.W., Mills, J.F., Bourne, M.C., Neely, A.D., Richards, A.H., Gregory, M.J. (1998). Testing manufacturing strategy formulation processes, International Journal of Production Economics, Vol. 56-57, 517-523, doi: 10.1016/ S0925-5273(97)00134-5.

[20] Hallgren, M., Olhager, J. (2006). Quantification in manufacturing strategy: A methodology and illustration, International Journal of Production Economics, Vol. 104, No. 1, 113-124, doi: 10.1016/i.ijpe.2005.09.004.

[21] Hayes, R.H., Pisano, G.P. (1994). Beyond world-class: The new manufacturing strategy, Harvard Business Review, Vol. 72, No. 1, 77-86.

[22] Ward, P.T., Bickford, D.J., Leong, G.K. (1996). Configurations of manufacturing strategy, business strategy, environment and structure, Journal of Management, Vol. 22, No. 4, 597-626, doi: 10.1177/014920639602200404.

[23] Mani, M., Madan, J., Lee, J.H., Lyons, K., Gupta, S.K. (2012). Characterizing sustainability for manufacturing performance assessment, In: Proceedings of the ASME 2012 International Design Engineering Technical Conferences \& Computers and Information in Engineering Conference, Chicago, Illinois, USA, 1-10.

[24] Rashid, A., Asif, F.M.A., Krajnik, P., Nicolescu, C.M. (2013). Resource conservative manufacturing: An essential change in business and technology paradigm for sustainable manufacturing, Journal on Cleaner Production, Vol. 57, 166-177, doi: 10.1016/j.jclepro.2013.06.012.

[25] Hassine, H., Barkallah, M., Bellacicco, A., Louati, J., Riviere, A., Haddar, M. (2015). Multi objective optimization for sustainable manufacturing, application in turning, International Journal of Simulation Modelling, Vol. 14, No. 1, 98-109, doi: 10.2507/ijsimm14(1)9.292.

[26] Joung, C.B., Carrell, J., Sarkar, P., Feng, S.C. (2013). Categorization of indicators for sustainable manufacturing, Ecological Indicators, Vol. 24, 148-157, doi: 10.1016/j.ecolind.2012.05.030.

[27] Kovac, M. (2012). Comparison of foresights in the manufacturing research, Transfer Inovácií, Vol. 23, 284-288.

[28] Tsai, W.H., Chou, W.C. (2009). Selecting management systems for sustainable development in SMEs: A novel hybrid model based on DEMATEL, ANP, and ZOGP, Expert Systems with Applications, Vol. 36, No. 2, Part 1, 14441458, doi: 10.1016/i.eswa.2007.11.058.

[29] Seuring, S., Müller, M. (2008). From a literature review to a conceptual framework for sustainable supply chain management, Journal of Cleaner Production, Vol. 16, No. 15, 1699-1710, doi: 10.1016/i.jclepro.2008.04.020. 
[30] Adams, C.A., Frost, G.R. (2008). Integrating sustainability reporting into management practices, Accounting Forum, Vol. 32, No. 4, 288-302, doi: 10.1016/i.accfor.2008.05.002.

[31] Elkington, J. (1997). Cannibals with forks: The triple bottom line of 21st century business, Capstone Publishing, Oxford.

[32] Placet, M., Anderson, R., Fowler, K.M. (2005). Strategies for sustainability, Research Technology Management, Vol. 48, No. 5, 32-41.

[33] Baumgartner, R.J., Ebner, D. (2010). Corporate sustainability strategies: Sustainability profiles and maturity levels, Sustainable Development, Vol. 18, No. 2, 76-89, doi: 10.1002/sd.447.

[34] Kashmanian, R.M., Wells, R.P., Keenan, C. (2011). Corporate environmental sustainability strategy: Key elements, Journal of Corporate Citizenship, Vol. 2011, No. 44, 107-130, doi: 10.9774/GLEAF.4700.2011.wi.00008.

[35] Danciu, V. (2013). The sustainable company: New challenges and strategies for more sustainability, Theoretical and Applied Economics, Vol. 20, No. 9, 7-26.

[36] Ageron, B., Gunasekaran, A., Spalanzani, A. (2012). Sustainable supply chain management: An empirical study, International Journal of Production Economics, Vol. 140, No. 1, 168-182, doi: 10.1016/j.ijpe.2011.04.007.

[37] Gimenez, C., Sierra, V., Rodon, J. (2012). Sustainable operations: Their impact on the triple bottom line, International Journal of Production Economics, Vol. 140, No. 1, 149-159, doi: 10.1016/j.ijpe.2012.01.035.

[38] Yuan, C., Zhai, Q., Dornfeld, D. (2012). A three dimensional system approach for environmentally sustainable manufacturing, CIRP Annals - Manufacturing Technology, Vol. 61, No. 1, 39-42, doi: 10.1016/j.cirp.2012.03.105.

[39] De Brucker, K., Macharis, C., Verbeke, A. (2013). Multi-criteria analysis and the resolution of sustainable development dilemmas: A stakeholder management approach, European Journal of Operational Research, Vol. 224, No. 1, 122-131, doi: 10.1016/j.ejor.2012.02.021.

[40] Kronenberg, J., Bergier, T. (2012). Sustainable development in a transition economy: Business case studies from Poland, Journal of Cleaner Production, Vol. 26, 18-27, doi: 10.1016/i.jclepro.2011.12.010.

[41] Matos, S., Silvestre, B.S. (2013). Managing stakeholder relations when developing sustainable business models: The case of the Brazilian energy sector, Journal of Cleaner Production, Vol. 45, 61-73, doi: 10.1016/i.jclepro. 2012.04.023.

[42] Griffiths, A., Petrick, J.A. (2001). Corporate architectures for sustainability, International Journal of Operations \& Production Management, Vol. 21, No. 12, 1573-1585, doi: 10.1108/01443570110410919.

[43] Johansson, G., Winroth, M. (2010). Introducing environmental concern in manufacturing strategies: Implications for the decision criteria, Management Research Review, Vol. 33, No. 9, 877-899, doi: 10.1108/01409171011 $\underline{070305}$.

[44] Theyel, G., Hofmann, K. (2012). Stakeholder relations and sustainability practices of US small and medium-sized manufacturers, Management Research Review, Vol. 35, No. 12, 1110-1133, doi: 10.1108/01409171211281255.

[45] Perrini, F., Tencati, A. (2006). Sustainability and stakeholder management: The need for new corporate performance evaluation and reporting systems, Business Strategy and the Environment, Vol. 15, No. 5, 296-308, doi: $10.1002 /$ bse. 538 .

[46] Clemens, B., Bakstran, L. (2010). A framework of theoretical lenses and strategic purposes to describe relationships among firm environmental strategy, financial performance, and environmental performance, Management Research Review, Vol. 33, No. 4, 393-405, doi: 10.1108/01409171011030480.

[47] De Brito, M.P., Carbone, V., Blanquart, C.M. (2008). Towards a sustainable fashion retail supply chain in Europe: Organization and performance, International Journal of Production Economics, Vol. 114, No. 2, 534-553, doi: 10.1016/j.ijpe.2007.06.012.

[48] Cheung, M.S., Myers, M.B., Mentzer, J.T. (2011). The value of relational learning in global buyer-supplier exchanges: A dyadic perspective and test of the pie-sharing premise, Strategic Management Journal, Vol. 32, No. 10, 1061-1082, doi: 10.1002/smj.926.

[49] Harrison, J.S., Bosse, D.A., Phillips, R.A. (2010). Managing for stakeholders, stakeholder utility functions, and competitive advantage, Strategic Management Journal, Vol. 31, No. 1, 58-74, doi: 10.1002/smj.801.

[50] Zadeh, L.A. (1965). Fuzzy set, Information and Control, Vol. 18, No. 3, 338-353, doi: 10.1016/S0019-9958(65) 90241-X.

[51] Promentilla, M.A.B., Furuichi, T., Ishii, K., Tanikawa, N. (2008). A fuzzy analytic network process for multi-criteria evaluation of contaminated site remedial countermeasures, Journal of Environmental Management, Vol. 88, No. 3, 479-495, doi: 10.1016/j.jenvman.2007.03.013.

[52] Tseng, M.L. (2009). A causal and effect decision-making model of service quality expectation using grey-fuzzy DEMATEL approach, Expert Systems with Applications, Vol. 36, No. 4, 7738-7748., doi: 10.1016/i.eswa.2008. 09.011.

[53] Wang, R.C., Chuu, S.J. (2004). Group decision-making using a fuzzy linguistic approach for evaluating the flexibility in a manufacturing system, European Journal of Operational Research, Vol. 154, No. 3, 563-572, doi: 10.1016/S0377-2217(02)00729-4.

[54] Von Altrock, C. (1996). Practical fuzzy-logic design, The Computer Applications Journal, Vol. 75, 1-5.

[55] Wang, C.H., Lu, I.Y., Chen, C.B. (2008). Evaluating firm technological innovation capability under uncertainty, Technovation, Vol. 28, No. 6, 349-363, doi: 10.1016/j.technovation.2007.10.007.

[56] Ocampo, L.A., Clark, E.E. (2014). A framework for capturing uncertainty of group decision-making in the context of the AHP/ANP, Advances in Industrial Engineering and Management, Vol. 3, No. 3, 7-16, doi: 10.7508/AIEM-V3N3-7-16.

[57] Opricovic, S., Tzeng, G.H. (2003). Defuzzification within a multi-criteria decision model, International Journal of Uncertainty, Fuzziness and Knowledge-Based Systems, Vol. 11, No. 5, 635-652, doi: 10.1142/S0218488503 $\underline{002387 .}$ 
[58] Tseng, M.L., Lin, Y.H., Chiu, A.S.F., Liao, J.C.H. (2008). Using FANP approach on selection of competitive priorities based on cleaner production implementation: A case study in PCB manufacturer, Taiwan, Clean Technologies and Environmental Policy, Vol. 10, No. 1, 17-29, doi: 10.1007/s10098-007-0109-4.

[59] Saaty, T.L. (2013). The analytic hierarchy process without the theory of Oskar Perron, International Journal of the Analytic Hierarchy Process, Vol. 5, No. 2, 268-293, doi: 10.13033/ijahp.v5i2.191.

[60] Saaty, T.L. (1980). The Analytic Hierarchy Process, McGraw-Hill, New York, USA.

[61] Smith, L., Ball, P. (2012). Steps towards sustainable manufacturing through modelling material, energy and waste flows, International Journal of Production Economics, Vol. 140, No. 1, 227-238, doi: 10.1016/j.ijpe.2012. $\underline{01.036}$.

[62] Chiacchio, M.S. (2011). Early impact assessment for sustainable development of enabling technologies, Total Quality Management and Excellence, Vol. 39, No. 3, 1-6. 